Discussion Paper No. 631

\title{
CHARACTERIZING THE VICKREY COMBINATORIAL AUCTION BY INDUCTION
}

\author{
Chew Soo Hong \\ and
}

Serizawa Shigehiro

March 2005

The Institute of Social and Economic Research Osaka University

6-1 Mihogaoka, Ibaraki, Osaka 567-0047, Japan 


\title{
Characterizing the Vickrey Combinatorial Auction by Induction ${ }^{1}$
}

\author{
Chew Soo Hong \\ Department of Economics, Hong Kong University of Science and Technology \\ Kowloon, Clearwater Bay, Hong Kong. \\ E-mail: shchew@ust.hk \\ AND \\ SERIZAWA SHIGEHIRO \\ Institute of Social and Economic Research, Osaka University \\ 6-1, Mihogaoka, Ibaraki, Osaka 567-0047, JAPAN \\ E-mail: serizawa@iser.osaka-u.ac.jp \\ Phone: (+81) 6-6879-8558 \Fax: $(+81)$ 6-6878-2766
}

March 3, 2005

This note studies the allocation of heterogeneous commodities to agents whose private values for combinations of these commodities are monotonic by inclusion. This setting can accommodate the presence of complementarity and substitutability among the heterogeneous commodities. By using induction logic, we provide an elementary proof of Holmstrom's (1919) characterization of the Vickrey combinatorial auction as the unique efficient, strategy-proof, and individually rational allocation rule. Our proof method can also be applied to domains to which his proof cannot be.

\footnotetext{
${ }^{1}$ Serizawa greatly benefited from discussion with Rajat Deb.
} 


\section{INTRODUCTION}

Recently, governments have increasingly been making use of auction mechanisms to allocate state-owned assets which exhibit significant extent of complementarity or substitutability. Examples of such assets include land, housing, spectrum rights (see, MacMillan, 1994), electricity transmission (see McCabe, Rassenti, and Smith, 1991), railroad tracks (Brewer and Plott, 1996), and space and time slots (Rassenti, Smith, and Bulfin, 1985; Banks, Ledyard, and Porter, 1989). The general problem concerns the allocation of heterogeneous commodities to agents with private values for combinations of these commodities. In this regard, one auction mechanism that has received much attention is the Vickrey combinatorial auction, which simultaneously allocates combinations of the commodities so that the total valuation is maximized for the reported bid profiles; and each agent is required to pay the social opportunity cost of allocating to him the combination of commodities he receives under the auction. ${ }^{2}$

An allocation rule is generally formulated as a function on the set of possible commodity value function profiles. Given an allocation rule, since agents' private valuations of commodities are not known to the others, there may be incentives for agents to misrepresent their values in order to manipulate the final outcomes to their favor. As a result, the actual outcomes may not constitute an efficient allocation relative to agents' true values. Thus it is advantageous for a rule to be immune to such strategic mispresentation and still attain an efficient allocation. If a rule is immune to strategic behavior by any agent, that is, if it is a dominant strategy for each agent to announce his true commodity values, then the rule is said to be strategy-proof. To attract a high number of agents, it is desirable for an allocation rule to exhibit the property of individual rationality, i.e., by never assigning any allocation which makes some agent worse off than he would be if he receives no commodity and pays nothing. Thus it is important to know what allocation rules are strategy-proof, individually rational, and efficient. It turns out that an answer can be found in Holmstrom (1979). He studies allocation rules in public goods model. His main results say that in public goods model, when the class of admissible preference profiles is convex, ${ }^{3}$ an allocation rule is strategy-proof and maximizes the net total benefit if and only if it is a Groves rule. ${ }^{4}$ In the case where the class of admissible preferences is convex, his results can also be applied to auction models to conclude that an allocation rule satisfying efficiency, strategy-proofness, and individual rationality is unique and can be implemented by the Vickrey combinatorial auction. ${ }^{5}$

Many authors disscussed the importance of the above characterization of the Vickrey combinatorial auction. ${ }^{6}$ However, there exists economic situations where the classes

\footnotetext{
${ }^{2}$ This auction is described in Vickrey (1961) for multiple homogeneous commodities. It coincides with the second-price auction when there is a single auction object. The Vickrey combinatorial auction is essentially the same as the influential Vickrey-Clarke-Groves mechanisms proposed by Clarke (1971) and Groves (1973). See Milgrom (2004) for the details.

${ }^{3}$ A class of preference profiles is "convex" if for any two value functions in the class of value functions, any convex combination of the two functions also in the class.

"In fact, a milder assumption of "the smooth connectedness" of the class of admissible preference profiles is sufficient.

${ }^{5}$ Similar characterizations for public good economies are previously established by Green and Laffont (1977), and Walker (1978). However, the characterizations of the these two articles cannot be applied to auction models since they assume that the class of admissible preferences include preferences which are not admissible in auction models.

${ }^{6}$ See Milgrom (2004) for example.
} 
of admissible value function profiles are not convex, that is, the above characerization cannnot be applied. By using mathematical induction, we prove the same conclusion on non-convex classes of value function profiles. This new proof enables us to apply the characterization to different situations. Besides, Holmstrom's (1979) proof employs advanced mathematical techniques. In view of the significance of Holmstrom's results for the efficient allocation of heterogenous commodities, it seems desirable to have a more accessible and easily understandable proof. By using mathematical induction, we dispense with the advanced mathematical techniques Holmstrom employs.

Section 2 sets up our model and states the main results formally. Section 3 contains concluding remarks. Proofs are presented in the Appendix.

\section{The Model and Main Results}

We denote the set of agents by $N=\{1,2, \ldots, n\}(n \geq 2)$. There is a set of commodities $M=\{1,2, \ldots, m\}(m \leq n)$. Let $\mathcal{M}=2^{M}$. A feasible commodity allocation $A=$ $\left(A^{1}, \ldots, A^{n}\right)$ is a partition of the set of commodities; that is, $A^{i} \cap A^{j}=\emptyset$ for any $i \in N$ and any $j \in N$ with $i \neq j$, and $\cup_{i \in N} A^{i}=M$. We denote the set of feasible commodity allocations by $\mathcal{A}$.

We denote the money agent $i$ pays by $t^{i} \in \mathbb{R}_{+}$. A feasible allocation is a pair $z=$ $(A ; t) \in \mathcal{A} \times \mathbb{R}_{+}^{n}$, where $t=\left(t^{1}, \ldots, t^{n}\right)$. We denote the set of feasible allocations by $\mathcal{Z}$. Agents have quasi-linear utility functions on $\mathcal{M} \times \mathbb{R}_{+}$. That is, each agent $i$ has a value function $v^{i}: \mathcal{M} \rightarrow \mathbb{R}_{+}$on commodity combinations; when agent $i$ receives $A^{i}$ and pays $t^{i} \in \mathbb{R}_{+}$, his utility is $u^{i}\left(A^{i}, t^{i}\right)=v^{i}\left(A^{i}\right)-t^{i}$. We normalize valuation functions so that $v^{i}(\emptyset)=0$. We consider the following types of value functions.

Definition 1: (i) A value function $v^{i}$ is monotonic if $v^{i}\left(A^{i}\right) \leq v^{i}\left(B^{i}\right)$ for any $A^{i}$ and $B^{i}$ with $A^{i} \subseteq B^{i}$;

(ii) it is strictly monotonic if $v^{i}\left(A^{i}\right)<v^{i}\left(B^{i}\right)$ for any $A^{i}$ and $B^{i}$ with $A^{i} \varsubsetneqq B^{i}$;

(iii) it is additive if $v^{i}\left(A^{i} \cup B^{i}\right)=v^{i}\left(A^{i}\right)+v^{i}\left(B^{i}\right)$ for any $A^{i} \subseteq M$ and $B^{i} \subseteq M$ with $A^{i} \cap B^{i}=\emptyset$;

(iv) it is superadditive if $v^{i}\left(A^{i} \cup B^{i}\right) \geq v^{i}\left(A^{i}\right)+v^{i}\left(B^{i}\right)$ for any $A^{i} \subseteq M$ and $B^{i} \subseteq M$ with $A^{i} \cap B^{i}=\emptyset$;

(v) it is subadditive if $v^{i}\left(A^{i} \cup B^{i}\right) \leq v^{i}\left(A^{i}\right)+v^{i}\left(B^{i}\right)$ for any $A^{i} \subseteq M$ and $B^{i} \subseteq M$ with $A^{i} \cap B^{i}=\emptyset$;

(vi) it exhibits homogeneity if $v^{i}\left(A^{i}\right)=v^{i}\left(B^{i}\right)$ for any $A^{i} \subseteq M$ and $B^{i} \subseteq M$ with $\# A^{i}=\# B^{i}$.

The conditions of subadditivity and superadditivity capture respectively the properties of substitutability and complementarity. For a value function which exhibits homogeneity, being subadditive (resp: superadditive) is equivalent to having nonincreasing (resp: nondecreasing) marginal utility. We denote the class of monotonic value functions by $V_{m}^{i}$, the class of strictly monotonic value functions by $V_{s m}^{i}$, the class of monotonic and additive value functions by $V_{a}^{i}$, and the class of strictly monotonic and additive value functions by $V_{s a}^{i}$.

Let $V^{i}$ denote the class of agent $i$ 's admissible value functions, and $V=V^{1} \times \cdots \times V^{n}$. A value function profile is an element of $V$. We call $V$ a class of value function profiles, or a domain for short. Given $N^{\prime} \subseteq N$, let $V^{N^{\prime}}=\prod_{j \in N^{\prime}} V^{j}$. We denote generic elements 
of $V, V^{N^{\prime}}$ and $V^{-i}$ by $v, v^{N^{\prime}}$ and $v^{-i}$ respectively. If $v=\left(v^{1}, \ldots, v^{n}\right) \in V, N^{\prime} \subseteq N$, and $i \in N$ are given in advance, $v^{N^{\prime}}$ denotes $\left(v^{j}\right)_{j \in N^{\prime}}$ and $v^{-i}$ denotes $\left(v^{j}\right)_{j \in N \backslash\{i\}}$.

Let $V_{m}=V_{m}^{1} \times \cdots \times V_{m}^{n}, V_{s m}=V_{s m}^{1} \times \cdots \times V_{s m}^{n}, V_{a}=V_{a}^{1} \times \cdots \times V_{a}^{n}$, and $V_{s a}=$ $V_{s a}^{1} \times \cdots \times V_{s a}^{n}$.

Definition 2: Given a class $V$ of value function profiles, an (allocation) rule on $V$ is a function $f$ from $V$ to $\mathcal{Z}$.

Given a rule $f: V \rightarrow \mathcal{Z}$ and $v \in V$, we denote agent $i$ 's outcome commodity set by $f_{A}^{i}(v)$, and his outcome payment by $f_{t}^{i}(v)$, and we write:

$f(v)=\left(f_{A}^{1}(v), \ldots, f_{A}^{n}(v) ; f_{t}^{1}(v), \ldots, f_{t}^{n}(v)\right), \quad f^{i}(v)=\left(f_{A}^{i}(v), f_{t}^{i}(v)\right), \quad f^{-i}(u)=f(v)_{j \neq i}$.

Definition 3: A commodity allocation $A \in \mathcal{A}$ is efficient for $v \in V$ if

$$
\sum_{i \in N} v^{i}(A)=\max \left\{\sum_{i \in N} v^{i}(B): B \in \mathcal{A}\right\} .
$$

An allocation $z$ is efficient for $v$ if its commodity allocation is efficient for $v$. A rule $f$ is efficient if for any $v \in V, f(v)$ is efficient for $v$.

Definition 4: A rule $f$ is strategy-proof if for any $v \in V$, any $i \in N$, and any $\hat{v}^{i} \in V^{i}$,

$$
u^{i}\left(f^{i}(v)\right) \geq u^{i}\left(f^{i}\left(\hat{v}^{i}, v^{-i}\right)\right)
$$

Definition 5: A rule $f: V \rightarrow \mathcal{Z}$ is individually rational if for any $v \in V$ and any $i \in N$,

$$
u^{i}\left(f^{i}(v)\right) \geq u^{i}(\emptyset, 0)=v^{i}(\emptyset)=0 .
$$

Definition 6: The Vickrey rule is a rule $g$ such that for any $v \in V$,

$$
g_{A}(v) \in \arg \max \left\{\sum_{i \in N} v^{i}(B): B \in \mathcal{A}\right\}
$$

and, $g_{t}^{i}(v)=\bar{\sigma}^{-i}(v)-\sigma^{-i}(v)$ for any $i \in N$, where

$$
\sigma^{-i}(v)=\sum_{j \neq i} v^{j}\left(g_{A}^{j}(v)\right) \text { and } \bar{\sigma}^{-i}(v)=\max \left\{\sum_{j \neq i} v^{j}(B): B \in \mathcal{A}\right\} .
$$

A Vickrey allocation rule is not unique, since the commodity allocation that maximizes the total value in (1) may not be unique.

REMARK 1: Let $g$ and $\hat{g}$ be Vickrey rules. Then

(i) $\sum_{i \in N} g_{t}^{i}(v)=\sum_{i \in N} \hat{g}_{t}^{i}(v)$ for any $v \in V$.

(ii) $u^{i}\left(g^{i}(v)\right)=u^{i}\left(\hat{g}^{i}(v)\right)$ for any $v \in V$ and any $i \in N$.

This remark follows since the efficiency of the Vickrey rules implies:

$$
\sum_{i \in N} v^{i}\left(g_{t}^{i}(v)\right)=\sum_{i \in N} v^{i}\left(\hat{g}_{t}^{i}(v)\right), \forall v \in V
$$

In view of the above remark, we may treat a Vickrey allocation rule as if it is unique. The Vickrey combinatorial auction is the direct mechanism for the Vickrey rule. ${ }^{7}$

\footnotetext{
${ }^{7} \mathrm{~A}$ mechanism is a pair consisting of an action space $S$ and an outcome function $h$, where $S=$ $S^{1} \times \cdots \times S^{n}, S^{i}$ is agent $i$ 's action space, and $h$ is a function from $S$ to $\mathcal{Z}$. The direct mechanism of an allocation rule $f$ on $V$ is the mechanism $(S, h)$ such that $S^{i}=V^{i}$ for each $i \in N$, and $h(v)=f(v)$ for all $v \in V$.
} 
The following theorems characterize the Vickrey rule as the unique allocation rule satisfying efficiency, strategy-proofness, and individual rationality. By definition, the Vickrey combinatorial auction implements it in dominant strategies. ${ }^{8}$

Definition 7 (Holmstrom 1979): A domain is $V=V^{1} \times \cdots \times V^{n}$ is smoothly connected if for any $i \in N$, any $v^{-i} \in V^{-i}$, any $v^{i} \in V^{i}$, and any $\widehat{v}^{i} \in V^{i}$, there exists a one-dimensional parameterized family of valuation functions in $V^{i}$ :

$$
V^{i}\left(v^{i}, \widehat{v}^{i}\right)=\left\{v^{i}(\cdot ; y) \in V^{i}: y \in[0,1]\right\}
$$

such that for all $A^{i}$,

$$
\text { (i) } v^{i}\left(A^{i} ; 0\right)=v^{i}\left(A^{i}\right), \quad \text { (ii) } v^{i}\left(A^{i} ; 1\right)=\widehat{v}^{i}\left(A^{i}\right), \quad \text { (iii) } \forall y \in[0,1], \exists \frac{\partial v^{i}\left(A^{i} ; 1\right)}{\partial y}
$$

and, moreover, for all $y \in[0,1]$ and all $A=\left(A^{i}, A^{-i}\right) \in A^{*}\left(v^{i}, \widehat{v}^{i} ; v^{-i}\right)$, where

$$
A^{*}\left(v^{i}, \widehat{v}^{i} ; v^{-i}\right)=\left\{A^{\prime} \in \mathcal{A}: \exists y \in[0,1] \text { such that } \sum_{i \in N} v^{i}\left(A^{i \prime}\right) \in \max \left\{\sum_{i \in N} v^{i}(B): B \in \mathcal{A}\right\}\right\}
$$

we have

$$
\text { (iv) } \exists K \in(0, \infty) \text { such that }\left|\frac{\partial v^{i}\left(A^{i} ; 1\right)}{\partial y}\right| \leq K .
$$

Definition 8: A domain is $V=V^{1} \times \cdots \times V^{n}$ is convex if for any $i \in N$, any $v^{i} \in V^{i}$, any $\widehat{v}^{i} \in V^{i}$, and any $y \in[0,1], y \cdot v^{i}+(1-y) \cdot \widehat{v}^{i} \in V^{i}$.

Examples of convex domain are the class $V_{m}$ of monotonic value functions, the class $V_{s m}$ of strictly monotonic value functions, the class $V_{a}$ of monotonic and additive value functions, the class $V_{s a}$ of strictly monotonic and additive value functions, the class of monotonic and superadditive value functions, the class of monotonic and subadditive value functions, etc. Holmstrom (1979) shows that the convexity of domain implies smooth connectedness. Thus, the classes mentioned above are also smoothly connected. Theorem 1 below can be applied to those classes of value functions.

Theorem 1 (Holmstrom 1979): Let $V$ be a smoothly connected domain. The Vickrey rule is the unique allocation rule that is efficient, strategy-proof, and individually rational on $V$.

Alothough Theorem 1 can be applied to various classes of value functions, there are also exist economic situations to which Theoremem 1 cannot be applied. For example, assume that bidders are firms who utilize the auctioned items to make profits. In this case, value functions represent technology of utilizing the items. If no technology exists in the industry that generates synergy effect among the items, and if no externality exists among them, a natural domain of allocation rules is the clsss $V_{a}$ of additive value functions. However, if there also exists a finite number of technologies $\bar{v}_{k}^{0}, k=1, \ldots, K$, in the society that generate synergy effect among items, but whether a firm can access such technologies or not is only known to the firm itself, then the domian is $V=V_{a}^{1} \cup\left\{\bar{v}_{k}^{0}: k=\right.$ $1, \ldots, K\} \times \cdots \times V_{a}^{n} \cup\left\{\bar{v}_{k}^{0}: k=1, \ldots, K\right\}$. This domain is not smoothly connected, and

\footnotetext{
${ }^{8} \mathrm{~A}$ mechanism $(S, h)$ implements a rule $f: V \rightarrow Z$ in dominant strategies if there is a dominant strategy $s^{i}: V^{i} \rightarrow S^{i}$ for each agent $i \in N$ and $h(s(v))=f(v)$ for all $v \in V$, where $s(v)=\left(s^{1}\left(v^{1}\right), \ldots, s^{n}\left(v^{n}\right)\right)$.
} 
so Holstrom's result cannot be applied. However, our results, Theorems 2 and 3, below can be applied.

TheOREm 2: Let $V$ be a domain such that $V_{s a} \subseteq V \subseteq V_{s m}$. The Vickrey rule is the unique allocation rule that is efficient, strategy-proof, and individually rational on $V$.

TheOREm 3: Let $V$ be a domain such that $V_{a} \subseteq V \subseteq V_{m}$. The Vickrey rule is the unique allocation rule that is efficient, strategy-proof, and individually rational on $V$.

See Appendix for the proofs. In the appendix, we formally prove Theorem 2 only since we can prove Theorem 3 similarly. We also show in Appendix that the conclusions of Theorems 2 hold when we replace the class of strictly monotonic value functions respectively by the other four classes of value functions in Definition 1: (ii) the class of strictly monotonic and additively value functions, (iii) the class of strictly monotonic and superadditive value functions, (iv) the class of strictly monotonic and subadditive value functions, and (v) the class of strictly monotonic and value functions exhibiting homogeneity. In addition, we can show that these results apply to the class of value functions exhibiting homogeneity and nonincreasing marginal utility. The idea of how to demonstrate the conclusions on these classes of value functions is discussed in the Appendix.

\section{Concluding Remarks}

In this note, we provide an alternative proof of Holmstrom's (1979) characterization of the Vickrey combinatorial auction as the unique efficient, strategy-proof, and individually rational allocation rule. While Holmstrom's proof method employes real analysis, ours employs induction logic. Moreover, our proof can be applied to nonconvex domains, to which Holmstrom's proof cannot be. Therefore, we have extended the situation in which the characterization of the Vickrey combinatorial auction holds.

\section{APPENDIX}

Proof of Theorem 2: Efficiency and individual rationality of the Vickrey rule follow directly from its definition. The usual argument implies that the Vickrey rule is strategyproof as well.

It remains to establish the uniqueness of a strategy-proof, efficient, and individually rational rule on the class of strictly monotonic value functions. Let $f$ be a rule that is strategy-proof, efficient, and individually rational on $V$. We prove that $f$ is the Vickrey rule. It follows directly from efficiency that

$$
f_{A}(v) \in \arg \max \left\{\sum_{i \in N} v^{i}\left(B^{i}\right): B \in \mathcal{A}\right\}
$$

for any $v \in V$. It suffices to prove $f_{t}^{i}(v)=\bar{\sigma}^{-i}(v)-\sigma^{-i}(v)$ for any $i \in N$ and any $v \in V$. Given $A^{i} \subseteq M$, we denote

$$
\bar{\sigma}^{-i}\left(v ; A^{i}\right)=\max \left\{\sum_{j \neq i} v^{j}\left(B^{i}\right): B \in \mathcal{A} \text { and } B^{i}=A^{i}\right\} .
$$

We employ the following facts. 
Fact 1: For any $v \in V$ and any $i \in N, f_{t}^{i}(v) \leq v^{i}\left(f_{A}^{i}(v)\right)$.

Fact 2: For any $v \in V$, any $i \in N$, and any $\hat{v}^{i} \in V^{i}$, if $f_{A}^{i}\left(\hat{v}^{i}, v^{-i}\right)=f_{A}^{i}(v)$, then $f_{t}^{i}\left(\hat{v}^{i}, v^{-i}\right)=f_{t}^{i}(v)$.

Fact 3: For any $v \in V$, any $i \in N$, and any $A \in \mathcal{A}$, if $A^{i}=f_{A}^{i}(v)$,

$$
\sum_{j \neq i} v^{j}\left(A^{j}\right) \leq \bar{\sigma}^{-i}\left(v ; A^{i}\right)=\sigma^{-i}(v) .
$$

Fact 1 follows from individual rationality. Fact 2 follows from strategy-proofness. In Fact 3 , the inequality follows from the definition of $\bar{\sigma}^{-i}\left(v ; A^{i}\right)$ and the equality follows from efficiency.

Let $v \in V$ and $i \in N$. We shall show by induction on the cardinality $\# f_{A}^{i}(v)$ of $f_{A}^{i}(v)$ that $f_{t}^{i}(v)=\bar{\sigma}^{-i}(v)-\sigma^{-i}(v)$. When $\# f_{A}^{i}(v)=0$, Fact 1 implies $f_{t}^{i}(v)=g_{t}^{i}(v)=0$. Thus, we start with the case of $\# f_{A}^{i}(v)=1$.

STEP 1: Assume that $\# f_{A}^{i}(v)=1$. Without loss of generality, let $f_{A}^{i}(v)=\{1\}$. Suppose $f_{t}^{i}(v) \neq \bar{\sigma}^{-i}(v)-\sigma^{-i}(v)$. We derive a contradiction in each of the following three cases.

Case 1: $f_{t}^{i}(v)>v^{i}\left(f_{A}^{i}(v)\right)$. This contradicts Fact 1 .

Case 2: $f_{t}^{i}(v)<\bar{\sigma}^{-i}(v)-\sigma^{-i}(v)$. Let $\hat{v}^{i} \in V_{s a}^{i}$ be such that

$$
f_{t}^{i}(v)<\hat{v}^{i}(\{1\})<\bar{\sigma}^{-i}(v)-\sigma^{-i}(v),
$$

and

$$
\hat{v}^{i}(\{k\})<\min _{j \neq i} \min _{A^{j} \subseteq M \backslash\{k\}}\left\{v^{j}\left(A^{j} \cup\{k\}\right)-v^{j}\left(A^{j}\right)\right\}, \forall k \neq 1 .
$$

We show $f_{A}^{i}\left(\hat{v}^{i}, v^{-i}\right)=\emptyset$. Together with (3), efficiency implies that $k \notin f_{A}^{i}\left(\hat{v}^{i}, v^{-i}\right)$ for any $k \neq 1$. Thus, $f_{A}^{i}\left(\hat{v}^{i}, v^{-i}\right)$ equals $\emptyset$ or $\{1\}$. For any $A \in \mathcal{A}$, if $A^{i}=\{1\}$, then since Fact 3 implies $\sum_{j \neq i} v^{j}\left(A^{j}\right) \leq \sigma^{-i}(v)$, it follows from the RHS of (2) that

$$
\hat{v}^{i}\left(A^{i}\right)+\sum_{j \neq i} v^{j}\left(A^{j}\right) \leq \hat{v}^{i}(\{1\})+\sigma^{-i}(v)<\bar{\sigma}^{-i}(v)
$$

Thus, any commodity allocation $A$ with $A^{i}=\{1\}$ is not efficient for $\left(\hat{v}^{i}, v^{-i}\right)$. Therefore, efficiency implies that $f_{A}\left(\hat{v}^{i}, v^{-i}\right)=\emptyset$.

Fact 1 , in conjunction with $f_{A}\left(\hat{v}^{i}, v^{-i}\right)=\emptyset$, implies that $f_{t}\left(\hat{v}^{i}, v^{-i}\right)=0$. It follows from the LHS of (2) that

$$
\hat{u}^{i}\left(f^{i}(v)\right)=\hat{v}^{i}(\{1\})-f_{t}^{i}(v)>0=\hat{u}^{i}\left(f^{i}\left(\hat{v}^{i}, v^{-i}\right)\right) .
$$

This contradicts strategy-proofness.

Case 3: $\bar{\sigma}^{-i}(v)-\sigma^{-i}(v)<f_{t}^{i}(v) \leq v^{i}\left(f_{A}^{i}(v)\right)$. Let $\hat{v}^{i} \in V_{s a}^{i}$ be such that

$$
\bar{\sigma}^{-i}(v)-\sigma^{-i}(v)<\hat{v}^{i}(\{1\})<f_{t}^{i}(v),
$$

and

$$
\hat{v}^{i}(\{k\})<\min _{j \neq i} \min _{A^{j} \subseteq M \backslash\{k\}}\left\{v^{j}\left(A^{j} \cup\{k\}\right)-v^{j}\left(A^{j}\right)\right\}, \forall k \neq 1 .
$$


We show $f_{A}^{i}\left(\hat{v}^{i}, v^{-i}\right)=\{1\}$. Together with (5), efficiency implies that $k \notin f_{A}^{i}\left(\hat{v}^{i}, v^{-i}\right)$ for any $k \neq 1$. Thus, $f_{A}^{i}\left(\hat{v}^{i}, v^{-i}\right)$ equals $\emptyset$ or $\{1\}$. For any $A \in \mathcal{A}$, if $A^{i}=\emptyset$, then it follows from the LHS of (4) that

$$
\hat{v}^{i}\left(A^{i}\right)+\sum_{j \neq i} v^{j}\left(A^{j}\right)=\leq \bar{\sigma}^{-i}(v)<\hat{v}^{i}(\{1\})+\sigma^{-i}(v)=\hat{v}^{i}\left(f_{A}^{i}(v)\right)+\sum_{j \neq i} v^{j}\left(f_{A}^{j}(v)\right) .
$$

Thus, any commodity allocation $A$ with $A^{i}=\emptyset$ is not efficient for $\left(\hat{v}^{i}, v^{-i}\right)$. Therefore, efficiency implies $f_{A}^{i}\left(\hat{v}^{i}, v^{-i}\right)=\{1\}$.

Fact 2 , in conjunction with $f_{A}^{i}\left(\hat{v}^{i}, v^{-i}\right)=\{1\}$ and the RHS of (4), implies that

$$
f_{t}^{i}\left(\hat{v}^{i}, v^{-i}\right)=f_{t}^{i}(v)>\hat{v}^{i}\left(f_{A}^{i}\left(\hat{v}^{i}, v^{-i}\right)\right) .
$$

This contradicts Fact 1.

STEP 2: Let $m^{\prime} \leq m$. As induction hypothesis, assume that if $\# f_{A}^{i}(v) \leq m^{\prime}-1$, then $f_{t}^{i}(v)=\bar{\sigma}^{-i}(v)-\sigma^{-i}(v)$. We show that if $\# f_{A}^{i}(v)=m^{\prime}$, then $f_{t}^{i}(v)=\bar{\sigma}^{-i}(v)-\sigma^{-i}(v)$. Without loss of generality, let $f_{A}^{i}(v)=\left\{1, \ldots, m^{\prime}\right\}$. Then Fact 3 implies that

$$
\sum_{j \neq i} v^{j}\left(A^{j}\right) \leq \sigma^{-i}\left(v ;\left\{1, \ldots, m^{\prime}\right\}\right)=\sigma^{-i}(v), \forall A \in \mathcal{A} \quad \text { э } A^{i}=\left\{1, \ldots, m^{\prime}\right\} .
$$

Suppose $f_{t}^{i}(v) \neq \bar{\sigma}^{-i}(v)-\sigma^{-i}(v)$. We derive a contradiction in each of the following two cases.

Case 1: $f_{t}^{i}(v)<\bar{\sigma}^{-i}(v)-\sigma^{-i}(v)$. Note

$$
f_{t}^{i}(v)+\bar{\sigma}^{-i}\left(v ;\left\{1, \ldots, m^{\prime}-1\right\}\right)-\bar{\sigma}^{-i}(v)<\bar{\sigma}^{-i}\left(v ;\left\{1, \ldots, m^{\prime}-1\right\}\right)-\sigma^{-i}(v) .
$$

Let $\hat{v}^{i} \in V_{s a}^{i}$ be such that

$$
\begin{gathered}
f_{t}^{i}(v)+\bar{\sigma}^{-i}\left(v ;\left\{1, \ldots, m^{\prime}-1\right\}\right)-\bar{\sigma}^{-i}(v)<\hat{v}^{i}\left(m^{\prime}\right)<\bar{\sigma}^{-i}\left(v ;\left\{1, \ldots, m^{\prime}-1\right\}\right)-\sigma^{-i}(v), \\
\hat{v}^{i}(\{k\})>\bar{\sigma}^{-i}(v), \forall k \in\left\{1, \ldots, m^{\prime}-1\right\}
\end{gathered}
$$

and

$$
\hat{v}^{i}(\{k\})<\min _{j \neq i} \min _{A^{j} \subseteq M \backslash\{k\}}\left\{v^{j}\left(A^{j} \cup\{k\}\right)-v^{j}\left(A^{j}\right)\right\}, \forall k \notin\left\{1, \ldots, m^{\prime}\right\} .
$$

We show here that $f_{A}^{i}\left(\hat{v}^{i}, v^{-i}\right)=\left\{1, \ldots, m^{\prime}-1\right\}$. Together with (9), efficiency implies that $k \notin f_{A}^{i}\left(\hat{v}^{i}, v^{-i}\right)$ for any $k \notin\left\{1, \ldots, m^{\prime}\right\}$, so that $f_{A}^{i}\left(\hat{v}^{i}, v^{-i}\right) \subseteq\left\{1, \ldots, m^{\prime}\right\}$. Together with (8), efficiency also implies $k \in f_{A}^{i}\left(\hat{v}^{i}, v^{-i}\right)$ for any $k \in\left\{1, \ldots, m^{\prime}-1\right\}$. Thus, $f_{A}^{i}\left(\hat{v}^{i}, v^{-i}\right)$ equals $\left\{1, \ldots, m^{\prime}-1\right\}$ or $\left\{1, \ldots, m^{\prime}\right\}$. Moreover, for any $A \in \mathcal{A}$, it follows from (6), the RHS of (7), and $\hat{v}^{i} \in V_{s a}^{i}$ that if $A^{i}=\left\{1, \ldots, m^{\prime}\right\}$, then

$$
\begin{aligned}
\hat{v}^{i}\left(A^{i}\right)+\sum_{j \neq i} v^{j}\left(A^{j}\right) & \leq \sum_{k=1}^{m^{\prime}} \hat{v}^{i}(\{k\})+\bar{\sigma}^{-i}\left(v ;\left\{1, \ldots, m^{\prime}\right\}\right) \\
& <\sum_{k=1}^{m^{\prime}-1} \hat{v}^{i}(\{k\})+\bar{\sigma}^{-i}\left(v ;\left\{1, \ldots, m^{\prime}-1\right\}\right) .
\end{aligned}
$$


Thus, any commodity allocation $A$ with $A^{i}=\left\{1, \ldots, m^{\prime}\right\}$ is not efficient for $\left(\hat{v}^{i}, v^{-i}\right)$. Therefore, efficiency implies that $f_{A}^{i}\left(\hat{v}^{i}, v^{-i}\right)=\left\{1, \ldots, m^{\prime}-1\right\}$.

The induction hypothesis, in conjunction with $f_{A}^{i}\left(\hat{v}^{i}, v^{-i}\right)=\left\{1, \ldots, m^{\prime}-1\right\}$ implies that

$$
\hat{u}^{i}\left(f^{i}\left(\hat{v}^{i}, v^{-i}\right)\right)=\sum_{k=1}^{m^{\prime}-1} \hat{v}^{i}(\{k\})-\left[\bar{\sigma}^{-i}(v)-\bar{\sigma}^{-i}\left(v ;\left\{1, \ldots, m^{\prime}-1\right\}\right)\right] .
$$

On the other hand, it follows from the LHS of (7) that

$$
\hat{u}^{i}\left(f^{i}(v)\right)>\sum_{k=1}^{m^{\prime}-1} \hat{v}^{i}(\{k\})-\left[\bar{\sigma}^{-i}(v)-\bar{\sigma}^{-i}\left(v ;\left\{1, \ldots, m^{\prime}-1\right\}\right)\right]=\hat{u}^{i}\left(f^{i}\left(\hat{v}^{i}, v^{-i}\right)\right) .
$$

This contradicts strategy-proofness.

Case 2: $f_{t}^{i}(v)>\bar{\sigma}^{-i}(v)-\sigma^{-i}(v)$. Note

$$
f_{t}^{i}(v)+\bar{\sigma}^{-i}\left(v ;\left\{1, \ldots, m^{\prime}-1\right\}\right)-\bar{\sigma}^{-i}(v)>\bar{\sigma}^{-i}\left(v ;\left\{1, \ldots, m^{\prime}-1\right\}\right)-\sigma^{-i}(v) .
$$

Let $\hat{v}^{i} \in V_{s a}^{i}$ be such that

$$
\begin{gathered}
f_{t}^{i}(v)+\bar{\sigma}^{-i}\left(v ;\left\{1, \ldots, m^{\prime}-1\right\}\right)-\bar{\sigma}^{-i}(v)>\hat{v}^{i}\left(\left\{m^{\prime}\right\}\right)>\bar{\sigma}^{-i}\left(v ;\left\{1, \ldots, m^{\prime}-1\right\}\right)-\sigma^{-i}(v), \\
\hat{v}^{i}(\{k\})>\bar{\sigma}^{-i}(v), \forall k \in\left\{1, \ldots, m^{\prime}-1\right\}
\end{gathered}
$$

and,

$$
\hat{v}^{i}(\{k\})<\min _{j \neq i} \min _{A^{j} \subseteq M \backslash\{k\}}\left\{v^{j}\left(A^{j} \cup\{k\}\right)-v^{j}\left(A^{j}\right)\right\}, \forall k \notin\left\{1, \ldots, m^{\prime}\right\} .
$$

We show here that $f_{A}^{i}\left(\hat{v}^{i}, v^{-i}\right)=\left\{1, \ldots, m^{\prime}\right\}$. Together with (12), efficiency implies that $k \notin f_{A}^{i}\left(\hat{v}^{i}, v^{-i}\right)$ for any $k \notin\left\{1, \ldots, m^{\prime}\right\}$, so that $f_{A}^{i}\left(\hat{v}^{i}, v^{-i}\right) \subseteq\left\{1, \ldots, m^{\prime}\right\}$. Together with (11), efficiency also implies that $k \in f_{A}^{i}\left(\hat{v}^{i}, v^{-i}\right)$ for any $k \in\left\{1, \ldots, m^{\prime}-1\right\}$. Thus, $f_{A}^{i}\left(\hat{v}^{i}, v^{-i}\right)$ equals $\left\{1, \ldots, m^{\prime}-1\right\}$ or $\left\{1, \ldots, m^{\prime}\right\}$. Moreover, for any $A \in \mathcal{A}$, it follows from (6), the RHS of (10), and $\hat{v}^{i} \in V_{s a}^{i}$ that if $A^{i}=\left\{1, \ldots, m^{\prime}-1\right\}$, then

$$
\begin{aligned}
\hat{v}^{i}\left(A^{i}\right)+\sum_{j \neq i} v^{j}\left(A^{j}\right) & \leq \sum_{k=1}^{m^{\prime}-1} \hat{v}^{i}(\{k\})+\bar{\sigma}^{-i}\left(v ;\left\{1, \ldots, m^{\prime}-1\right\}\right) \\
& <\sum_{k=1}^{m^{\prime}} \hat{v}^{i}(\{k\})+\bar{\sigma}^{-i}\left(v ;\left\{1, \ldots, m^{\prime}\right\}\right) .
\end{aligned}
$$

Thus, any commodity allocation $A$ with $A^{i}=\left\{1, \ldots, m^{\prime}-1\right\}$ is not efficient for $\left(\hat{v}^{i}, v^{-i}\right)$. Therefore, efficiency implies that $f_{A}^{i}\left(\hat{v}^{i}, v^{-i}\right)=\left\{1, \ldots, m^{\prime}\right\}$.

Fact 2 , in conjunction with $f_{A}^{i}\left(\hat{v}^{i}, v^{-i}\right)=\left\{1, \ldots, m^{\prime}\right\}$ implies that $f_{t}^{i}\left(\hat{v}^{i}, v^{-i}\right)=f_{t}^{i}(v)$. Therefore,

$$
\hat{u}^{i}\left(f^{i}\left(\hat{v}^{i}, v^{-i}\right)\right)=\sum_{k=1}^{m^{\prime}} \hat{v}^{i}(\{k\})-f_{t}^{i}(v)
$$

Let $\widetilde{v}^{i} \in V_{s a}^{i}$ be such that

$$
\widetilde{v}^{i}(\{k\})>\bar{\sigma}^{-i}(v), \forall k \in\left\{1, \ldots, m^{\prime}-1\right\},
$$


and

$$
\widetilde{v}^{i}(\{k\})<\min _{j \neq i} \min _{A^{j} \subseteq M \backslash\{k\}}\left\{v^{j}\left(A^{j} \cup\{k\}\right)-v^{j}\left(A^{j}\right)\right\}, \forall k \notin\left\{1, \ldots, m^{\prime}-1\right\} .
$$

Then together with (14), efficiency implies that $k \notin f_{A}^{i}\left(\widetilde{v}^{i}, v^{-i}\right)$ for any $k \notin\left\{1, \ldots, m^{\prime}-1\right\}$, so that $f_{A}^{i}\left(\widetilde{v}^{i}, v^{-i}\right) \subseteq\left\{1, \ldots, m^{\prime}-1\right\}$. Together with (13), efficiency also implies that $k \in f_{A}^{i}\left(\widetilde{v}^{i}, v^{-i}\right)$ for any $k \in\left\{1, \ldots, m^{\prime}-1\right\}$. Thus, $f_{A}^{i}\left(\widetilde{v}^{i}, v^{-i}\right)=\left\{1, \ldots, m^{\prime}-1\right\}$, so that

$$
f_{t}^{i}\left(\widetilde{v}^{i}, v^{-i}\right)=\bar{\sigma}^{-i}(v)-\bar{\sigma}^{-i}\left(v ;\left\{1, \ldots, m^{\prime}-1\right\}\right),
$$

by the induction hypothesis. It follows from the LHS of (10) and $\hat{v}^{i} \in V_{s a}^{i}$ that

$$
\begin{aligned}
\hat{u}^{i}\left(f^{i}\left(\widetilde{v}^{i}, v^{-i}\right)\right) & =\sum_{k=1}^{m^{\prime}-1} \hat{v}^{i}(\{k\})-\left[\bar{\sigma}^{-i}(v)-\bar{\sigma}^{-i}\left(v ;\left\{1, \ldots, m^{\prime}-1\right\}\right)\right] \\
& >\sum_{k=1}^{m^{\prime}-1} \hat{v}^{i}(\{k\})-\left[\left(f_{t}^{i}(v)-\hat{v}^{i}\left(m^{\prime}\right)\right]\right. \\
& =\sum_{k=1}^{m^{\prime}} \hat{v}^{i}(\{k\})-f_{t}^{i}(v) \\
& =\hat{u}^{i}\left(f^{i}\left(\hat{v}^{i}, v^{-i}\right)\right) .
\end{aligned}
$$

This contradicts strategy-proofness.

We have established the uniqueness of the strategy-proof, efficient, and individually rational allocation rule on the class of strictly monotonic value functions.

Here, we show how the result of Theorem 2 can be proved on the class of strictly monotonic and additive value functions (denoted by $V_{s a}$ ), the class of strictly monotonic and subadditive value functions (denoted by $V_{s b}$ ), the class of strictly monotonic and superadditive value functions (denoted by $V_{s p}$ ), the class of strictly monotonic value functions exhibiting homogeneity (denoted by $V_{s h}$ ), and the class of strictly monotonic value functions exhibiting homogeneity with nonincreasing marginal utility (denoted by $V_{s d}$ ).

Note that Facts 1, 2, and 3 hold on these five classes of value functions. In the proof of Theorem 2 , besides the original value profile $v$ being selected from $V_{s m}$, we use candidate value functions $\hat{v}^{i}$ and $\widetilde{v}^{i}$ from $V_{s a}$, to derive contradictions in Cases 2 and 3 of Step 1 and Cases 1 and 2 of Step 2. It is apparent that the above method of proof can be applied to demonstrate the uniqueness on $V_{s a}, V_{s b}$, and $V_{s p}$, each of which contains $V_{s a}$. For $V_{s h}$, and $V_{s d}$, the present method of proof applies with slight modifications. That is, in deriving the contradictions in Cases 2 and 3 of Step 1 and Cases 1 and 2 of Step 2, we can choose the candidate value functions $\hat{v}^{i}$ and $\widetilde{v}^{i}$ from $V_{s h}$ and $V_{s d}$, respectively.

\section{References}

[1] Banks, Jeffrey S., John O. Ledyard, and David P. Porter, "Allocating Uncertain and Unresponsive Resources: An Experimental Approach," RAND Journal of Economics (1989): 1-25 
[2] Brewer, Paul J. and Charles R. Plott, "A Binary Conflict Ascending Price (BICAP) Mechanism for the Decentralized Allocation of the Right to use Railroad Tracks," International Journal of Industrial Organization (1996): 857-886

[3] Clarke, Edward H., "Multipart Pricing of Public Goods," Public Choice (1971): 1733

[4] Green, Jerry, and Lafont, Jean-Jacques, "Characterization of Satisfactory Mechanisms For the Revelation of Preferences for Public Goods," Econometrica (1977): 427-438

[5] Groves, Theodore, "Incentives in Teams," Econometrica (1973): 617-632

[6] Holmstrom, Bengt, "Groves' Scheme on Restricted Domain," Econometrica (1979): $1137-1144$

[7] MacMillan, John, "Selling Spectrum Rights," Journal of Economic Perspectives (1994): 145-162

[8] McCabe, Kevin A., Stephen J. Rassenti, and Vernon L. Smith., "Auction Institutional Design: Theory and Behavior of Simultaneous Multiple-unit Generalizations of the Dutch and English Auctions," American Economic Review (1991): 1276-83

[9] Milgrom, Paul, "Putting Auction Theory to Work: The Simultaneous Ascending Auction," Journal of Political Economy (2000): 245-272

[10] Milgrom, Paul, "Putting Auction Theory to Work," (2004) Cambridge University Press.

[11] Rassenti, Steve, Vernon L. Smith, and R.L. Bulfin, "A Combinatorial Auction Mechanism for Airport Time Slot Allocation," Bell Journal of Economics (1985): 402-417

[12] Vickrey, William, Counterspeculation, auctions, and competitive sealed tenders, Journal of Finance (1961): 8-37

[13] Walker, Mark, "A Note on the Characterization of Mechanisms for the Revalation of Preferences," Econometrica (1978): 147-152 